\title{
Add-on Therapy of EPA Reduces Oxidative Stress and Inhibits the Progression of Aortic Stiffness in Patients with Coronary Artery Disease and Statin Therapy: A Randomized Controlled Study
}

\author{
Akira Takaki $^{1,2}$, Seiji Umemoto ${ }^{3}$, Kaoru Ono ${ }^{4,5}$, Kouzaburo Seki ${ }^{6}$, Tsutomu Ryoke ${ }^{7,8}$, Akihisa Fujii ${ }^{6,9}$, \\ Tatsunori Itagaki ${ }^{10}$, Masahiko Harada ${ }^{11}$, Masakazu Tanaka ${ }^{12,13}$, Takahito Yonezawa ${ }^{14}$, Hiroshi Ogawa \\ and Masunori Matsuzaki ${ }^{15}$ for the ELIA study group \\ ${ }^{1}$ Department of Cardiology, Tokuyama Central Hospital, Shunan, Japan \\ ${ }^{2}$ Takaki Medical Clinic, Shunan, Japan \\ ${ }^{3}$ Pharmaceutical Clinical Research Center, Yamaguchi University Hospital, Ube, Japan \\ ${ }^{4}$ Department of Cardiology, Shunan City Shinnanyou Hospital, Shunan, Japan \\ ${ }^{5}$ Ono Clinic, Shunan, Japan \\ ${ }^{6}$ Department of Cardiology, Yamaguchi Rosai Hospital, Sanyo Onoda, Japan \\ ${ }^{7}$ Department of Cardiology, Shimonoseki Kosei Hospital, Shimonoseki, Japan \\ ${ }^{8}$ Ryoke Cardiology Clinic, Shimonoseki, Japan \\ ${ }^{9}$ Mitsuyama Clinic, Hofu, Japan \\ ${ }^{10}$ Department of Cardiology, Hikari Municipal Yamato General Hospital, Hikari, Japan \\ ${ }^{11}$ Department of Cardiology, Ube Industries Central Hospital, Ube, Japan \\ ${ }^{12}$ Department of Cardiology, Shuto General Hospital, Yanai, Japan \\ ${ }^{13}$ Ishihara Internal Medicine and Cardiology Hospital, Fukuoka, Japan \\ ${ }^{14}$ Department of Cardiology, Souyou Hospital, Hofu, Japan \\ ${ }^{15}$ Department of Medicine and Clinical Science, Yamaguchi University Graduate School of Medicine, Ube, Japan
}

\begin{abstract}
Aim: We examined the anti-oxidant mechanisms of combined therapy of eicosapentaenoic acid (EPA) plus statin on the progression of atherosclerosis.

Methods: Patients receiving statin therapy for dyslipidemia and with coronary artery disease (CAD) were assigned randomly in an open-label manner to the EPA $(1,800 \mathrm{mg} /$ day $)$-plus-statin group ( $n=25$; combined-therapy group) or to the statin-only group $(n=25)$, and followed for 48 weeks. At baseline and 48 weeks after enrollment, oxidative stress, brachial-ankle pulse wave velocity (baPWV) and stiffness parameter $\beta$-index of the carotid were measured.

Results: The lipid profile remained unchanged throughout the study. Although the median value of baPWV increased more in the statin-only group than in the combined-therapy group, this difference was not significant $(p=0.29)$; however, a decrease in baPWV was associated with combined-therapy treatment by multiple regression analysis adjusted for age and mean blood pressure $(p=0.04)$. In addition, the $\beta$-index of the carotid was lower in the combined-therapy group than in the statin-only group $(p=0.02)$. Furthermore, although the difference in the reduction of the urinary concentration of 8-isoprostane between the two groups did not reach statistical significance, this concentration was significantly lower in the combined-therapy group with higher baseline levels $(\geq 183 \mathrm{pg} / \mathrm{mL} \cdot \mathrm{Cr})$ of urinary 8-isoprostane $(p=0.004)$.

Conclusions: EPA may reduce oxidative stress and inhibit the progression of arterial stiffness more efficiently than statin-only therapy in patients with dyslipidemia and CAD.
\end{abstract}

\section{J Atheroscler Thromb, 2011; 18:857-866.}

Key words; Eicosapentaenoic acid, Oxidative stress, Arterial stiffness, Coronary artery disease

Address for correspondence: Seiji Umemoto, Pharmaceutical

Clinical Research Center, Yamaguchi University Hospital,

1-1-1 Minami Kogushi, Ube, Yamaguchi 755-8505 Japan

E-mail: umemoto@yamaguchi-u.ac.jp

Received: October 5, 2010

Accepted for publication: April 14, 2011

\section{Introduction}

HMG-CoA reductase inhibitor (statin) therapy reduces mortality and morbidity in patients with coronary artery disease $(\mathrm{CAD})^{1,2)}$; however, some trials 
have also highlighted the significance of residual cardiovascular risk after treatment of low density lipoprotein cholesterol (LDL-C) to target levels, which reflects the complex nature of residual cardiovascular risk $^{3)}$. Evidence for the cardioprotective nature of $\omega-3$ fatty acids, including eicosapentaenoic acid (EPA), a $\omega-3$ polyunsaturated fatty acid (PUFA), indicates that $\omega-3$ fatty acid therapy also shows great promise in the secondary prevention of CAD ${ }^{4-6)}$. According to recent studies, the addition of EPA to statin therapy provides further benefits in preventing cardiovascular events, mainly through cholesterol-independent mechanisms, in patients with hypercholesterolemia, especially for the secondary prevention of $\mathrm{CAD}^{7,8)}$.

Pulse wave velocity (PWV), a measure of large artery stiffness, is widely used as an indicator of arterial stiffness and as a marker reflecting vascular damage to diagnose arteriosclerosis, and is an important predictor of cardiovascular events ${ }^{9,10)}$. In addition, carotid intima-media thickness (IMT) and carotid plaque have been reported to be independent predictors of $\mathrm{CAD}^{11,12)}$. These noninvasive imaging modalities are useful surrogate markers for future cardiovascular events ${ }^{13)}$.

We previously reported that the amount of oxidative stress in the aorta might be an important factor leading to vascular dysfunction, as assessed by arterial stiffness ${ }^{14)}$, and that the decreased superoxide content of the aorta caused by lipid-lowering drugs through antioxidant mechanisms independent of lipid-lowering effects may inhibit the progression of atherosclerosis and arterial stiffness in persons with hypercholesterolemia ${ }^{15)}$, suggesting that oxidative stress may be one of the important determinants of arterial stiffness in hypercholesterolemia.

\section{Aim}

Although the results of a previous trial demonstrated the efficacy of EPA in preventing CAD in patients with hypercholesterolemia under statin therapy, especially for secondary prevention ${ }^{7}$, the precise mechanisms of this prevention, especially with regard to oxidative stress, in preventing the progression of atherosclerosis following cardiovascular events remain unknown. Thus, in the EPA Lipid Intervention and Atherosclerosis Prevention (ELIA) study, to clarify the anti-oxidant mechanisms of combined therapy of EPA plus statin on the progression of atherosclerosis, we examined the additional effects of EPA on oxidative stress, arterial stiffness and atherosclerosis in patients with CAD who were already receiving statins.

\section{Methods}

\section{Ethics Committee Procedure and Consent}

The study protocol was approved by the ethics committees of all involved institutions, and the study was undertaken in accordance with the Declaration of Helsinki and Ethics Guidelines for Clinical Studies in Japan. All of the study patients gave their fully informed written consent for study participation.

\section{Study Design and Patient Population}

The ELIA study was an investigator-initiated, multi-center study in accordance with the prospective, randomized open blinded endpoint evaluation (PROBE) design of once daily statin versus statin plus EPA (1,800 mg), involving individuals who had experienced cardiovascular disease with dyslipidemia.

Inclusion criteria were outpatient status, age over 20 years and less than 70 years, with dyslipidemia and a previous history of CAD (myocardial infarction, angina pectoris, coronary angioplasty or coronary artery bypass graft surgery) at least 3 months before entering the study, regardless of sex, with total cholesterol $\geq 180 \mathrm{mg} / \mathrm{dL}$ or LDL-C $\geq 100 \mathrm{mg} / \mathrm{dL}$ and taking any statin. Exclusion criteria for the study were 1) history of myocardial infarction, angina pectoris, coronary angioplasty or coronary artery bypass graft surgery within 3 months prior to enrollment in the study, 2) family history of hypercholesterolemia and secondary hypertension or dyslipidemia, 3) uncontrolled diabetes mellitus with $\mathrm{HbA} 1 \mathrm{c} \geq 8.0 \%$, 4) severe heart failure (New York Heart Association functional classification IV), 5) severe peripheral arterial disease (Fontaine Class II, III or IV), 6) chronic atrial fibrillation or flutter, 7) bleeding tendency, 8) serious liver dysfunction (aspartate aminotransferase or alanine aminotransferase $\geq 100 \mathrm{IU} / \mathrm{L}$ ) or serious renal dysfunction (serum creatinine $\geq 2 \mathrm{mg} / \mathrm{dL}$ ), 9) pregnancy, 10) history of malignancy 5 years prior to study entry, 11) compliance rate $<80 \%$ assessed by a patient interview, 12) other serious illness or significant abnormalities that the investigator judged inappropriate for inclusion in the study.

Eligible patients were randomly assigned to a study arm. Randomization was conducted at the University Hospital Medical Information Network (UMIN) Center, the University of Tokyo Hospital, by the dynamic allocation method (the modified minimization method) after stratification using diabetes and the smoking status as adjusting factors in the minimization calculation.

All of the study patients received a statin once a day; the choice of statin was entrusted to the investi- 
gator. The study population was randomly assigned to receive EPA with statin (combined-therapy group) or statin alone (statin-only group), and both groups were followed up for 48 weeks. In the combined-therapy group, we administered a daily dose of $1,800 \mathrm{mg}$ EPA. At each out-patient clinic visit, study doctors provided dietary instructions and monitored medication use, and a questionnaire was given to confirm the adherence to the study medications. Systolic and diastolic blood pressure levels and heart rates were measured twice after the patient had sat quietly for at least 5 minutes at approximately 2-minute intervals, and mean values were used for the study.

\section{Measurement of Arterial Stiffness and Atheroscle- rosis}

We used two different indices to more reliably evaluate arterial stiffness, the brachial-ankle PWV (baPWV) and the stiffness parameter $\beta$-index of the carotid, since the baPWV is known to be influenced by blood pressure ${ }^{9)}$, whereas the stiffness parameter $\beta$-index is a blood pressure-independent patient-specific arterial stiffness measured by ultrasound ${ }^{14,15)}$. The baPWV was automatically obtained using AT-form PWV/ABI (Omron Colin, Tokyo, Japan), which has been described in detail previously ${ }^{16-18)}$.

All of the study patients underwent ultrasonography of the carotid artery to assess IMT and the plaque score. Replicate scans were performed by qualified well-trained ultrasonographers at baseline and at 48 weeks to preserve the quality control of image acquisition. At baseline and the 48-week follow-up visit, a B-mode scan was performed with image acquisition at one predefined angle of the far wall of six carotid segments: the right and left common carotid arteries, carotid bifurcations, and internal carotid arteries. All images were transferred to the ultrasonography core laboratory. The stiffness parameter $\beta$-index, as measured by carotid ultrasound with a $7.5-\mathrm{MHz}$ transducer, was calculated at a point without plaque, as previously reported ${ }^{14,15,19)}$. IMT was evaluated as the distance between the lumen-intimal interface and the medial-adventitial interface, and was measured using two calipers on the frozen frame of a suitable longitudinal image. The upper normal limit for the IMT was defined as $1.0 \mathrm{~mm}$, and the plaque score was calculated by the sum of IMT $\geq 1.1 \mathrm{~mm}$. The max IMT of both carotids was used to evaluate atherosclerosis for the study. To assess the severity of atherosclerosis, we also used the plaque score, which was calculated by summing all plaque thickness in both carotid systems ${ }^{20}$. These indices obtained by carotid ultrasound were assessed by a specialist sonographer in a blind fashion.

\section{Laboratory Measurements}

All of the blood and urine samples were collected at the outpatient clinic from patients in a fasting state and were measured by a central laboratory (SRL, Inc., Tokyo, Japan). Serum cholesterol and triglyceride were measured enzymatic ally, high-density lipoprotein cholesterol (HDL-C) was measured by direct measurement, remnant-like particle cholesterol (RLP-C) was measured by immunoseparation assay ${ }^{21)}$, antioxidant enzyme superoxide dismutase (SOD) activity was measured by the nitrite method modified by Oyanagui $^{22)}$, malondialdehyde modified LDL (MDA-LDL), which is one of the surrogate markers for atherogenic oxidized LDL, was measured by enzyme-linked immunosorbent assay ${ }^{21)}$, urinary concentration of 8-isoprostane, one of the non-cyclooxygenase-derived prostanoids, was measured by enzyme immunoassay, high-sensitivity C-reactive protein (hs-CRP) was measured by the nephelometry method, and EPA and arachidonic acid (AA), a $\omega-6$ PUFA, were measured by gaschromatography, respectively.

\section{Statistical Analysis}

The Wilcoxon 2-sample test was used to compare continuous variables. The $\chi^{2}$ test was used to compare categorical variables. The change from baseline to 48 weeks after intervention was evaluated using the Wilcoxon signed rank test. Multiple regression analysis was used to evaluate the relations between EPA treatment and baPWV adjusted for age and mean blood pressure. All analyses were performed using version 5.0.1a of the JMP statistical software program (SAS Institute, Inc., Cary, NC, USA). Data are expressed as the mean \pm SD or median (interquartile range). Significance was established at $p<0.05$.

\section{Results}

\section{Patients}

From May 2004 until August 2005, 51 patients were enrolled in the study, and follow-up was continued until August 2006. Of these patients, 50 underwent randomization, with 25 assigned to the statinonly group and 25 assigned to the EPA-plus-statin (combined-therapy) group, respectively. We excluded one patient from the analysis because of missing baseline data.

Demographic and clinical characteristics of the patients are listed in Table 1. There were no significant differences in the baseline clinical characteristics of the patients, except that the statins chosen by the 
Table 1. Baseline characteristics of the study patients

\begin{tabular}{|c|c|c|c|}
\hline Characteristic & $\begin{array}{l}\text { Statin-only } \\
\quad(n=25)\end{array}$ & $\begin{array}{l}\text { Combined therapy } \\
\qquad(n=25)\end{array}$ & $p$ value \\
\hline Age, mean (SD), y & $60.9(7.0)$ & $61.6(5.6)$ & 0.89 \\
\hline Male, No. (\%) & $20(80.0)$ & $21(84.0)$ & 0.71 \\
\hline BMI, mean (SD), $\mathrm{kg} / \mathrm{m}^{2}$ & $24.0(3.6)$ & $25.1(2.3)$ & 0.12 \\
\hline \multicolumn{4}{|l|}{ Coronary artery disease, No. (\%) } \\
\hline OMI & $12(48.0)$ & $16(64.0)$ & 0.39 \\
\hline $\mathrm{AP}$ & $10(40.0)$ & $8(32.0)$ & 0.56 \\
\hline CABG & $4(16.0)$ & $3(12.0)$ & 0.68 \\
\hline PCI & $12(48.0)$ & $13(52.0)$ & 0.78 \\
\hline NYHA class, No. & & & 0.57 \\
\hline $\mathrm{I}$ & 23 & 24 & \\
\hline II & 1 & 1 & \\
\hline III & 1 & 0 & \\
\hline Diabetes, No. (\%) & $8(32.0)$ & $7(28.0)$ & 0.76 \\
\hline Hypertension, No. (\%) & $16(64.0)$ & $14(56.0)$ & 0.56 \\
\hline Current smoker, No. (\%) & $6(24.0)$ & $5(20.0)$ & 0.73 \\
\hline Statin, No. & 25 & 25 & 1.00 \\
\hline Pravastatin & 5 & 14 & \\
\hline Simvastatin & 1 & 2 & \\
\hline Fluvastatin & 4 & 2 & $(0.02)$ \\
\hline Atorvastatin & 13 & 4 & \\
\hline Pitavastatin & 1 & 3 & \\
\hline Antidiabetic agents, No. (\%) & $4(16.0)$ & $4(16.0)$ & 1.00 \\
\hline Sulfonylurea & 0 & 3 & $(0.04)$ \\
\hline Biguanides & 2 & 1 & $(0.55)$ \\
\hline$\alpha$-GI drug & 0 & 2 & $(0.09)$ \\
\hline Thiazolidine & 0 & 1 & $(0.24)$ \\
\hline Insulin & 4 & 0 & $(0.02)$ \\
\hline Antihypertensive agents, No. (\%) & $20(80.0)$ & $18(72.0)$ & 0.51 \\
\hline calcium antagonist & 17 & 7 & $(0.004)$ \\
\hline ACE inhibitor/angiotensin II antagonist & 14 & 16 & $(0.56)$ \\
\hline$\beta$-blocker & 6 & 4 & $(0.48)$ \\
\hline Diuretic & 2 & 1 & $(0.55)$ \\
\hline Others & 1 & 0 & $(0.24)$ \\
\hline Antiplatelet/anticoagulants, No. (\%) & $22(88.0)$ & $19(76.0)$ & 0.27 \\
\hline Aspirin & 20 & 19 & $(0.73)$ \\
\hline Ticlopidine & 6 & 2 & $(0.12)$ \\
\hline Cilostazol & 1 & 1 & $(1.00)$ \\
\hline Warfarin & 3 & 0 & $(0.04)$ \\
\hline
\end{tabular}

Abbreviations: OMI, old myocardial infarction; AP, angina pectoris; CABG, coronary artery bypass grafting; PCI, percutaneous coronary intervention; NYHA, New York Heart Association; $\alpha$-GI, $\alpha$-glucosidase inhibitor; ACE, angiotensin-converting enzyme. No. indicates number of patients. Parentheses indicate the results of the $\chi^{2}$ test. Statin was prescribed as a single drug in each patient. Concomitant drugs such as antidiabetic or antihypertensive agents and antiplatelet/anticoagulants were prescribed as combination therapy in some patients in both groups.

investigators were not the same for all patients; pravastatin was prescribed in the statin-only group, whereas atorvastatin was more preferably prescribed in the combined-therapy group, although all patients in both groups were prescribed a statin as standard therapy. Good adherence of the study patients to the study drug protocol (i.e., receipt of at least $80 \%$ of a study medication) was maintained throughout the study in 
Table 2. Levels of blood pressure, lipids, remnant like protein cholesterol, malondialdehyde modified low-density lipoprotein, urinary 8-isoprostane, and C-reactive protein in patients with coronary artery disease at baseline and after 48 weeks of treatment with study medications

\begin{tabular}{|c|c|c|c|c|c|}
\hline Characteristic & Group & Baseline & 48 weeks & $\begin{array}{l}\text { Amount of change } \\
\text { from baseline }\end{array}$ & $p$ value $^{\dagger}$ \\
\hline SBP, mmHg & Statin-only & $131 \pm 15$ & $128 \pm 17$ & 2.7 & 0.72 \\
\hline \multirow{2}{*}{ DBP, $\mathrm{mmHg}$} & Statin-only & $77 \pm 9$ & $74 \pm 9$ & 0.8 & \multirow{2}{*}{0.62} \\
\hline & Combined therapy & $76 \pm 7$ & $75 \pm 9$ & 1.3 & \\
\hline \multirow{2}{*}{ LDL-C, mg/dL } & Statin-only & $106 \pm 19$ & $109 \pm 32$ & 6.8 & \multirow{2}{*}{0.06} \\
\hline & Combined therapy & $110 \pm 23$ & $107 \pm 21$ & -5.1 & \\
\hline \multirow{2}{*}{ HDL-C, mg/dL } & Statin-only & $57 \pm 9$ & $61 \pm 14$ & 4.4 & \multirow{2}{*}{0.13} \\
\hline & Combined therapy & $52 \pm 11$ & $52 \pm 12$ & 0.1 & \\
\hline \multirow{2}{*}{ RLP-C, mg/dL } & Statin-only & $6.3 \pm 4.4$ & $6.8 \pm 9.2$ & 0.7 & \multirow{2}{*}{0.11} \\
\hline & Combined therapy & $4.3 \pm 5.4$ & $3.6 \pm 2.1$ & 0.6 & \\
\hline \multirow{2}{*}{ SOD activity, $\mathrm{U} / \mathrm{mL}$} & Statin-only & $3.0 \pm 1.9$ & $3.0 \pm 2.1$ & 0.2 & \multirow{2}{*}{0.66} \\
\hline & Combined therapy & $3.9 \pm 2.5$ & $4.2 \pm 3.4$ & 0.0 & \\
\hline \multirow{2}{*}{ MDA-LDL, U/L } & Statin-only & $117.0 \pm 63.5$ & $121.9 \pm 38.2$ & 5.0 & \multirow{2}{*}{0.71} \\
\hline & Combined therapy & $113.8 \pm 32.2$ & $118.0 \pm 28.1$ & 9.3 & \\
\hline \multirow{2}{*}{ Urinary 8-isoprostane, $\mathrm{pg} / \mathrm{mg} \cdot \mathrm{Cr}$} & Statin-only & $239 \pm 117$ & $219 \pm 120$ & -21.6 & \multirow{2}{*}{0.16} \\
\hline & Combined therapy & $246 \pm 198$ & $154 \pm 72$ & -100.1 & \\
\hline hs-CRP, ng/mL & Statin-only & $574 \pm 829$ & $956 \pm 1553$ & 508.6 & 0.03 \\
\hline
\end{tabular}

Abbreviations: SBP, systolic blood pressure; DBP, diastolic blood pressure; CHO, cholesterol; LDL-C, low-density lipoprotein cholesterol; HDL-C, high-density lipoprotein cholesterol; RLP-C, remnant like protein cholesterol; SOD, superoxide dismutase; MDA-LDL, malondialdehyde modified low-density lipoprotein; hs-CRP, high sentive C-reactive protein; $\mathrm{Cr}$, creatinine. Data are the mean \pm SD unless specified. IQR, interquartile range. ${ }^{\dagger} P$ values are for differences between the two treatment groups in the amount of change from baseline using the Wilcoxon signed rank test.

both groups.

\section{Laboratory Results}

The difference in EPA/AA ratio between the two groups was low at baseline, whereas the EPA/AA ratio in the combined-therapy group increased substantially from the baseline to 24 weeks after the administration of EPA compared with the statin-only group (statinonly group versus combined-therapy group: 0.69 \pm 0.38 versus $0.85 \pm 0.46$ at baseline, not significant, $0.69 \pm 0.39$ versus $1.57 \pm 0.72$ at 24 weeks, $p<0.0001$, respectively).

Table 2 summarizes the laboratory results in the ELIA study. There were few differences in the amount of change of blood pressure, total cholesterol, LDL-C, HDL-C, non HDL-C, triglyceride, RLP-C, SOD activity and MDA-LDL between the two groups, whereas the amount of change of hs-CRP was significantly less in the statin-only group than in the combined-therapy group. In addition, although the reduction of urinary concentration of 8-isoprostane was greater in the combined-therapy group than in the statin-only group, this difference did not reach statistical significance.

\section{Effects of Combined Therapy of EPA Plus Statin on Arterial Stiffness and Max IMT and Plaque Score of the Carotid Artery}

Table 3 summarizes the effects of EPA on the baPWV, $\beta$-index of the carotid, max IMT and plaque score of the carotid artery in CAD and statin-therapy patients. There was no difference in the amount of 
Table 3. Effects of adding EPA treatment to the statin therapy on baPWV, $\beta$-index of the carotid artery, max IMT and plaque score of the carotid artery in patients with coronary artery disease at baseline and after 48 weeks of the treatment of study medications

\begin{tabular}{|c|c|c|c|c|c|}
\hline Characteristic & Group & Baseline & 48 weeks & Amount of change from baseline & $p$ value $^{\dagger}$ \\
\hline \multirow{2}{*}{$\mathrm{baPWV}, \mathrm{cm} / \mathrm{sec}$} & Statin-only & $1615(1458-1816)$ & $1670(1585-1793)$ & 90.9 & \multirow{2}{*}{0.29} \\
\hline & Combined therapy & $1514(1332-1656)$ & $1552(1385-1684)$ & 44.1 & \\
\hline \multirow{2}{*}{ Carotid $\beta$-index } & Statin-only & $7.1(5.2-11.4)$ & $7.6(6.0-12.9)$ & -0.0 & \multirow{2}{*}{0.02} \\
\hline & Combined therapy & $9.4(5.6-12.4)$ & $5.5(4.5-8.1)$ & -2.7 & \\
\hline \multirow{2}{*}{ Max IMT, mm } & Statin-only & $1.0(0.9-1.3)$ & $1.1(0.9-1.5)$ & 0.06 & \multirow{2}{*}{0.50} \\
\hline & Combined therapy & $1.3(0.9-1.4)$ & $1.2(0.8-1.5)$ & 0.09 & \\
\hline \multirow{2}{*}{ Plaque score } & Statin-only & $1.3(0.8-6.3)$ & $1.0(0.0-4.2)$ & -1.1 & \multirow{2}{*}{0.60} \\
\hline & Combined therapy & $1.9(0.0-6.2)$ & $1.9(0.0-3.0)$ & -0.5 & \\
\hline
\end{tabular}

Abbreviations: baPWV, brachial-ankle pulse wave velocity; IMT, intima-media thickness. The baPWV was automatically obtained using AT-form PWV/ABI (Omron Colin, Tokyo, Japan). The stiffness parameter $\beta$-index as measured by carotid ultrasound was calculated at a point without plaque. Max IMT of the carotid artery was obtained by carotid ultrasound. Data are expressed as the median (interquartile range). ${ }^{\dagger} p$ values are for differences between the two treatment groups in the amount of change from baseline using the Wilcoxon signed rank test.

Table 4. Multiple regression analysis of the relationship between baPWV and other associated variables

\begin{tabular}{lrrrr}
\hline & $\beta$ & SE & $t$ value & $p$ value \\
\hline Combined therapy, yes/no & -75.4 & 34.7 & -2.17 & 0.04 \\
Age, years & 12.8 & 5.7 & 2.25 & 0.03 \\
Mean blood pressure, $\mathrm{mmHg}$ & 5.3 & 3.6 & 1.48 & 0.15 \\
\hline
\end{tabular}

Abbreviations: baPWV, brachial-ankle pulse wave velocity; $\beta$, standardized regression coefficients; SE, standard error. Combined-therapy; EPA (1,800 mg/day) plus statin. Mean blood pressure was calculated from diastolic blood pressure + [(systolic blood pressure-diastolic blood pressure)/3].

change in baPWV between the two groups, although the increase in the median value of the baPWV from baseline in the statin-only group was greater than that in the combined-therapy group. The decrease in the median value of the $\beta$-index of the carotid from baseline to 48 weeks was significantly lower in the combined-therapy group than in the statin-only group. There were no differences in the amount of change of the max IMT and plaque score between the two groups.

Furthermore, the results of multiple regression analysis adjusted for age and mean blood pressure showed a significant association between the decrease in baPWV and combined-therapy treatment (Table 4).

\section{Effects of Combined Therapy of EPA Plus Statin on Urinary Concentration of 8-Isoprostane}

To further explore the effect of EPA on oxidative stress, we examined the effect of EPA on urinary concentration of 8-isoprostane based upon the baseline urinary concentration of 8-isoprostane divided by the median value at $183 \mathrm{pg} / \mathrm{mL} \cdot \mathrm{Cr}$ (Fig. 1). There was little difference in both higher and lower urinary con- centrations of 8-isoprostane between the statin-only group and the combined-therapy group at baseline. Interestingly, the subgroup with a higher urinary concentration of 8-isoprostane in the combined-therapy group at 48 weeks was significantly lower than at baseline, and the concentration became significantly lower than at 48 weeks in the statin-only group. On the other hand, in the subgroups with a lower urinary concentration of 8-isoprostane, the concentration remained unchanged at 48 weeks compared with those values at baseline.

\section{Adverse Events}

After randomization, 1 patient in the combinedtherapy group and 1 patient in the statin-only group experienced nonfatal myocardial infarction and unstable angina pectoris, respectively. None of the patients involved in the study showed side effects of the study medications.

\section{Discussion}

Several lipid-independent mechanisms in addi- 

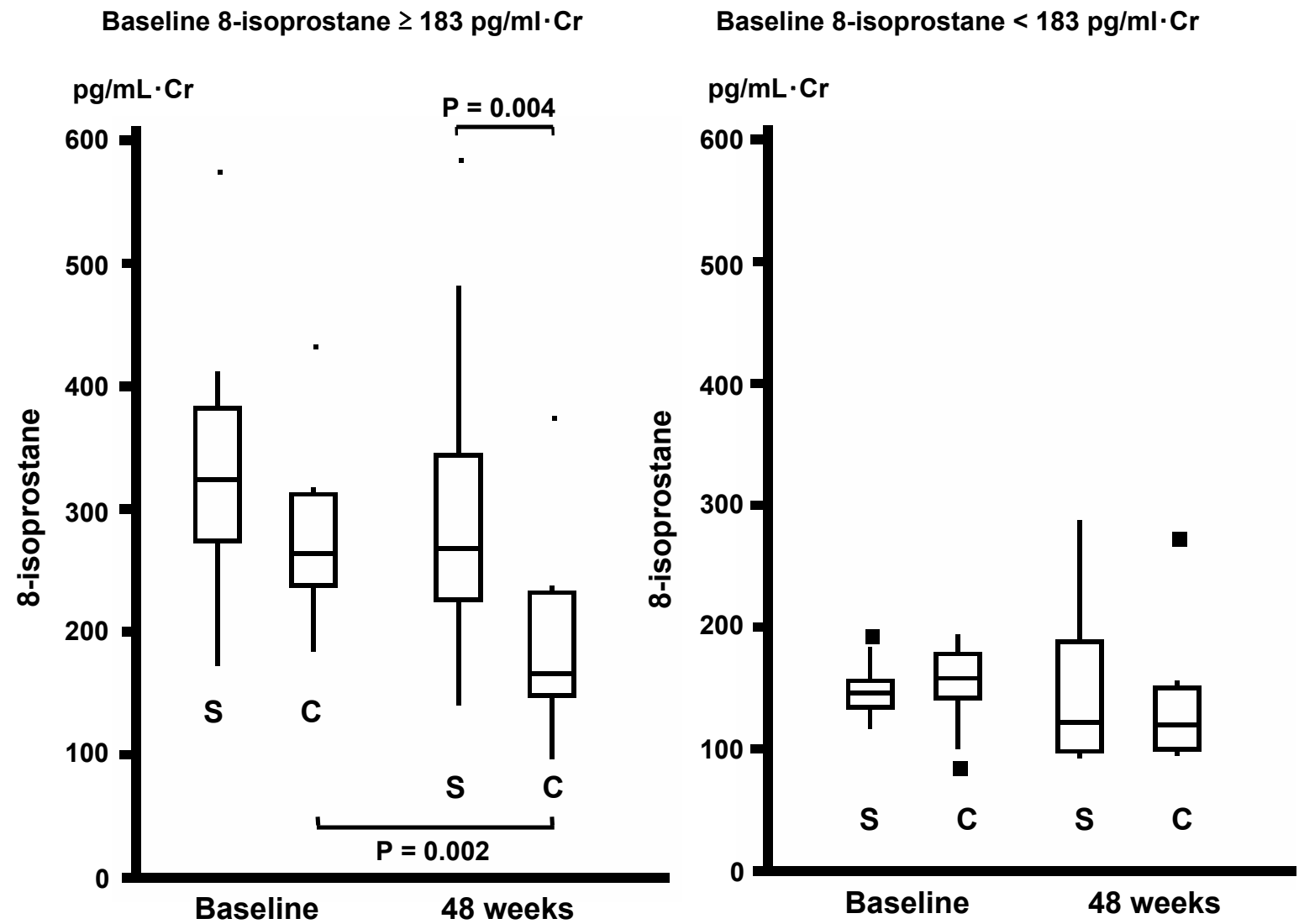

Fig. 1. Effects of adding EPA treatment to statin therapy on urinary concentration of 8-isoprostane in patients with coronary artery disease.

Each group was divided into two subgroups by the median value $(183 \mathrm{pg} / \mathrm{mL} \cdot \mathrm{Cr})$ based upon the baseline urinary concentration of 8-isoprostane. In subgroups with a higher urinary concentration of 8-isoprostane, add-on therapy of EPA induced a significant lowering effect on the concentration compared with the statin-only group. In contrast, there was little effect of EPA observed in the subgroups with lower urinary concentrations of 8-isoprostane. Data express the box and schematic plot. The line drawn across the box indicates the median. The lower line of the box is at the first quartile (Q1), and the upper line is at the third quartile (Q3). The whiskers are lines that extend from the top or bottom of the box to adjacent values within $\pm 1.5 \times(\mathrm{Q} 3-\mathrm{Q} 1)$. Closed square outlier. ${ }^{*} p=0.002$ versus baseline in the combined-therapy group. $\mathrm{S}$, statinonly group; $\mathrm{C}$, combined-therapy group.

tion to decreased triglycerides, including anti-inflammatory effects, modulation of autonomic function, vasodilation, decreased blood pressure and plaque stabilization, have been suggested to result from the association between $\omega-3$ PUFAs and the risk of cardiovascular disease ${ }^{4)}$. In the present study, we clearly demonstrated that combined EPA-plus-statin therapy was superior to statin-only therapy for inhibition of the progression of arterial stiffness independent of lipid lowering.

The study population in the present study showed good adherence to the study protocol. The EPA/AA ratio was significantly higher in the combined-therapy group than in the statin-only group. In previous studies, the EPA/AA ratio was significantly lower in CAD patients than in healthy volunteers, and platelet aggregation, serotonin release, the amount of thromboxane $\mathrm{B}_{2}$, a cyclooxygenase product synthesized from exogenous $\mathrm{AA}$, and insulin resistance were increased by decreasing the ratio of EPA to $\mathrm{AA}^{23,24)}$. In addition, the results of a previous study demonstrated that in good adherers with previous CAD, EPA substantially reduced the risk compared with statinonly therapy ${ }^{25)}$. These results suggest that the increase in the EPA/AA ratio in the present study may explain the favorable effects of EPA in the combined-therapy group.

It has been reported that baPWV is an index of arterial stiffness with similar characteristics to aortic PWV ${ }^{26)}$, and is a marker of severity of atherosclerotic vascular damage as well as a predictor of prognosis in patients with cardiovascular disease ${ }^{27)}$; however, PWV 
is strongly influenced by blood pressure and age ${ }^{9)}$. Blood pressures were unchanged throughout the study, and there was no difference in the amount of change in baPWV between the two groups, although the increase in the median baPWV value from baseline was greater in the statin-only group than in the combined-therapy group. However, multiple regression analysis adjusted for age and blood pressure clearly demonstrated an association between a decrease in baPWV and combined-therapy treatment, as shown in Table 4. The $\beta$-index of the carotid at 48 weeks was also significantly lower in the combined-therapy group than in the statin-only therapy group. Previous studies demonstrated that EPA supplements attenuated age-related increases in arterial stiffness in patients with dyslipidemia ${ }^{28)}$ and improved arterial stiffness and were less influenced by blood pressure in patients with metabolic syndrome ${ }^{29)}$. These results may support our findings regarding arterial stiffness, suggesting that EPA supplements attenuate age-related increases in arterial stiffness in patients with CAD and statin therapy.

Recent studies have suggested that excess oxidative stress induced by hyperlipidemia may play a critical role in the progression of atherosclerosis ${ }^{30,31)}$. We previously reported that arterial stiffness in patients with hypercholesterolemia may be preceded by arterial sclerosis ${ }^{32)}$, and the smooth muscle phenotype of the aortic tunica media may be one of the important determinant factors of aortic stiffness ${ }^{14,33)}$. Furthermore, statin inhibits the increase in oxidative stress and halts the progression of arterial stiffness independent of lipid lowering in vivo ${ }^{14,15)}$. In the present study, although the reduction of the urinary concentration of 8-isoprostane was greater in the combinedtherapy group than in the statin-only group, the difference between groups did not reach statistical significance. However, we clearly demonstrated a significant lowering effect of the urinary concentration of 8-isoprostane in combined-therapy group patients with a higher baseline concentration of urinary 8-isoprostane. The formation of 8-isoprostane in lipid bilayers may contribute to alterations in the fluidity and integrity of cellular membranes, inducing oxidant injury, and the level of 8-isoprostane reflects in vivo oxidative stress $^{34)}$, suggesting that the inhibitory effects of EPA on the observed changes in baPWV and the $\beta$-index of the carotid in the combined-therapy group might have been due to the modification of oxidative stress, mainly in patients with CAD and statin therapy with a higher oxidative stress condition, which may have caused functional changes in the vasculature by the addition of EPA to the statin therapy beyond lipid modification.

The levels of MDA-LDL and RLP-C in patients with CAD were higher than those in the control group, suggesting that the association between high levels of remnant lipoprotein cholesterol and high oxidized LDL levels might be linked to atherogenesis in patients with $\mathrm{CAD}^{21)}$. Nakamura et al. previously reported that the administration of EPA to patients with hyperlipidemia and statin therapy for about 30 months induced a significant decrease in serum triglyceride and RLP-C ${ }^{35)}$. In another study, treatment with a $\omega-3$ fatty acid for 4 weeks had no effect on hs-CRP levels or triglyceride levels in patients with $\mathrm{CAD}^{36)}$. In addition, after statin (pitavastatin) treatment for 12 months, significant decreases in 8-hydroxy- 2'-deoxyguanosine, which is a marker of oxidative DNA damage, MDA-LDL and aortic stiffness, were observed in type 2 diabetic patients, and the change of aortic stiffness significantly correlated with the change of MDA-LDL ${ }^{37)}$. In the present study, RLP-C, SOD activity and MDA-LDL remained unchanged by combined therapy compared with statin-only therapy. It is still unknown why the level of hs-CRP in the combined-therapy group was higher at both baseline and 48 weeks, and why there was a significant difference in the level hs-CRP between the two groups. Nonetheless, the high level of hs-CRP did not affect aortic stiffness based upon the results obtained in the study.

\section{Study Limitations}

First, the follow-up period of the present study was only 48 weeks. It is likely that more time is needed to see the effects of EPA in addition to statin therapy, since the prevention of cardiovascular events by the addition of EPA required more than 2 years ${ }^{7)}$. Our study demonstrated that the effect of EPA on aortic stiffness may precede the inhibition of cardiovascular events. Second, in this study, the choice and applied dosage of statin was entrusted to each investigator, and the study period was relatively short. These differences may have caused different results for these indices between the present study and others ${ }^{35-37)}$. Furthermore, the number and class of anti-diabetic and antihypertensive drugs, and antiplatelet/anticoagulants were different between the two groups, although these drugs were not changed throughout the study; we cannot exclude the possibility that this difference in drugs may have affected the results. In addition, the importance of central pressure and derived central hemodynamic indices compared with peripheral pressure for cardiovascular outcomes and all-cause mortal- 
ity was reported recently ${ }^{38)}$. Since in our study we used peripheral blood pressure to evaluate arterial stiffness, it is possible that we would have obtained different results had we used central blood pressure. Finally, this study had an exploratory and PROBE design, and the sample size was small, so the results of the study may have been influenced by these factors. Further study will be necessary to confirm our results.

\section{Conclusion}

Combined EPA-plus-statin therapy may reduce oxidative stress and inhibit the progression of arterial stiffness more efficiently than statin-only therapy, independent of cholesterol lowering in patients with dyslipidemia and a previous history of CAD.

\section{Acknowledgments}

We are indebted to all the study participants, to Drs. Eizo Akagawa, Ryo Hashimoto and Taketo Tanigawa, and the nurses, ultrasonographers and hospital staff for their commitment to the study, and to all the patients who participated in the ELIA study.

\section{Disclosure}

This work was supported in part by grants from the Ministry of Education, Culture, Sports, Science and Technology of Japan. Clinical trial registration information: UMIN-CTR UMIN000002171.

\section{References}

1) Wilt TJ, Bloomfield HE, MacDonald R, Nelson D, Rutks I, Ho M, Larsen G, McCall A, Pineros S, Sales A: Effectiveness of statin therapy in adults with coronary heart disease. Arch Intern Med, 2004; 164: 1427-1436

2) Baigent C, Keech A, Kearney PM, Blackwell L, Buck G, Pollicino C, Kirby A, Sourjina T, Peto R, Collins R, Simes R: Efficacy and safety of cholesterol-lowering treatment: Prospective meta-analysis of data from 90,056 participants in 14 randomised trials of statins. Lancet, 2005; 366: $1267-1278$

3) Sharma RK, Singh VN, Reddy HK: Thinking beyond low-density lipoprotein cholesterol: Strategies to further reduce cardiovascular risk. Vasc Health Risk Manag, 2009; 5: 793-799

4) Lee JH, O'Keefe JH, Lavie CJ, Harris WS: Omega-3 fatty acids: Cardiovascular benefits, sources and sustainability. Nat Rev Cardiol, 2009; 6: 753-758

5) Jacobson TA: Beyond lipids: The role of omega-3 fatty acids from fish oil in the prevention of coronary heart disease. Curr Atheroscler Rep, 2007; 9: 145-153

6) Lavie CJ, Milani RV, Mehra MR, Ventura HO: Omega-3 polyunsaturated fatty acids and cardiovascular diseases. J Am Coll Cardiol, 2009; 54: 585-594

7) Matsuzaki M, Yokoyama M, Saito Y, Origasa H, Ishikawa Y, Oikawa S, Sasaki J, Hishida H, Itakura H, Kita T, Kitabatake A, Nakaya N, Sakata T, Shimada K, Shirato K, Matsuzawa Y: Incremental effects of eicosapentaenoic acid on cardiovascular events in statin-treated patients with coronary artery disease. Circ J, 2009; 73: 1283-1290

8) Yokoyama M, Origasa H, Matsuzaki M, Matsuzawa Y, Saito Y, Ishikawa Y, Oikawa S, Sasaki J, Hishida H, Itakura H, Kita T, Kitabatake A, Nakaya N, Sakata T, Shimada K, Shirato K: Effects of eicosapentaenoic acid on major coronary events in hypercholesterolaemic patients (JELIS): A randomised open-label, blinded endpoint analysis. Lancet, 2007; 369: 1090-1098

9) Cecelja M, Chowienczyk P: Dissociation of aortic pulse wave velocity with risk factors for cardiovascular disease other than hypertension: A systematic review. Hypertension, 2009; 54: 1328-1336

10) Inoue $N$, Maeda $R$, Kawakami $H$, Shokawa $T$, Yamamoto $\mathrm{H}$, Ito C, Sasaki H: Aortic pulse wave velocity predicts cardiovascular mortality in middle-aged and elderly japanese men. Circ J, 2009; 73: 549-553

11) Simon A, Megnien JL, Chironi G: The value of carotid intima-media thickness for predicting cardiovascular risk. Arterioscler Thromb Vasc Biol, 2010; 30: 182-185

12) Lee E, Emoto M, Teramura M, Tsuchikura $S$, Ueno $H$, Shinohara K, Morioka T, Mori K, Koyama H, Shoji T, Okuno Y, Inaba M, Nishizawa Y: The combination of IMT and stiffness parameter beta is highly associated with concurrent coronary artery disease in type 2 diabetes. J Atheroscler Thromb, 2009; 16: 33-39

13) Feinstein SB, Voci P, Pizzuto F: Noninvasive surrogate markers of atherosclerosis. Am J Cardiol, 2002; 89: 31C-43C; discussion 43C-44C

14) Itoh $S$, Umemoto $S$, Hiromoto $M$, Toma $Y$, Tomochika $Y$, Aoyagi S, Tanaka M, Fujii T, Matsuzaki M: Importance of $\mathrm{NAD}(\mathrm{P}) \mathrm{H}$ oxidase-mediated oxidative stress and contractile type smooth muscle myosin heavy chain SM2 at the early stage of atherosclerosis. Circulation, 2002; 105: 2288-2295

15) Umeji K, Umemoto $S$, Itoh $S$, Tanaka $M$, Kawahara $S$, Fukai T, Matsuzaki M: Comparative effects of pitavastatin and probucol on oxidative stress, $\mathrm{Cu} / \mathrm{Zn}$ superoxide dismutase, PPAR-gamma, and aortic stiffness in hypercholesterolemia. Am J Physiol Heart Circ Physiol, 2006; 291: $\mathrm{H} 2522-2532$

16) Yamashina $A$, Tomiyama $H$, Takeda $K$, Tsuda $H$, Arai $T$, Hirose K, Koji Y, Hori S, Yamamoto Y: Validity, reproducibility, and clinical significance of noninvasive brachial-ankle pulse wave velocity measurement. Hypertens Res, 2002; 25: 359-364

17) Matsui Y, Kario K, Ishikawa J, Eguchi K, Hoshide S, Shimada K: Reproducibility of arterial stiffness indices (pulse wave velocity and augmentation index) simultaneously assessed by automated pulse wave analysis and their associated risk factors in essential hypertensive patients. Hypertens Res, 2004; 27: 851-857

18) Munakata M, Ito N, Nunokawa T, Yoshinaga K: Utility of automated brachial ankle pulse wave velocity measure- 
ments in hypertensive patients. Am J Hypertens, 2003; 16: 653-657

19) Hirai T, Sasayama S, Kawasaki T, Yagi S: Stiffness of systemic arteries in patients with myocardial infarction. A noninvasive method to predict severity of coronary atherosclerosis. Circulation, 1989; 80: 78-86

20) O'Leary DH, Polak JF, Kronmal RA, Manolio TA, Burke GL, Wolfson SK Jr: Carotid-artery intima and media thickness as a risk factor for myocardial infarction and stroke in older adults. Cardiovascular health study collaborative research group. N Engl J Med, 1999; 340: 14-22

21) Hiki M, Shimada K, Ohmura H, Kiyanagi T, Kume A, Sumiyoshi K, Fukao K, Inoue N, Mokuno H, Miyazaki T, Daida H: Serum levels of remnant lipoprotein cholesterol and oxidized low-density lipoprotein in patients with coronary artery disease. J Cardiol, 2009; 53: 108-116

22) Oyanagui Y: Reevaluation of assay methods and establishment of kit for superoxide dismutase activity. Anal Biochem, 1984; 142: 290-296

23) Hashimoto Y, Naito C, Kawamura M, Oka H: Effects of the ratio of exogenous eicosapentaenoic acid to arachidonic acid on platelet aggregation and serotonin release. Thromb Res, 1984; 34: 439-446

24) Yanagisawa N, Shimada K, Miyazaki T, Kume A, Kitamura Y, Ichikawa R, Ohmura H, Kiyanagi T, Hiki M, Fukao K, Sumiyoshi K, Hirose K, Matsumori R, Takizawa H, Fujii K, Mokuno H, Inoue N, Daida H: Polyunsaturated fatty acid levels of serum and red blood cells in apparently healthy japanese subjects living in an urban area. J Atheroscler Thromb, 2010; 17: 285-294

25) Origasa H, Yokoyama M, Matsuzaki M, Saito Y, Matsuzawa Y: Clinical importance of adherence to treatment with eicosapentaenoic acid by patients with hypercholesterolemia. Circ J, 2010; 74: 510-517

26) Tsuchikura S, Shoji T, Kimoto E, Shinohara K, Hatsuda S, Koyama H, Emoto M, Nishizawa Y: Brachial-ankle pulse wave velocity as an index of central arterial stiffness. J Atheroscler Thromb, 2010; 17: 658-665

27) Wang KL, Cheng HM, Sung SH, Chuang SY, Li CH, Spurgeon HA, Ting CT, Najjar SS, Lakatta EG, Yin FC, Chou P, Chen $\mathrm{CH}$ : Wave reflection and arterial stiffness in the prediction of 15-year all-cause and cardiovascular mortalities: A community-based study. Hypertension, 2010; 55: 799-805

28) Tomiyama H, Takazawa K, Osa S, Hirose K, Hirai A, Iketani T, Monden M, Sanoyama K, Yamashina A: Do eicosapentaenoic acid supplements attenuate age-related increases in arterial stiffness in patients with dyslipidemia?: A preliminary study. Hypertens Res, 2005; 28: 651-655

29) Satoh N, Shimatsu A, Kotani K, Himeno A, Majima T, Yamada K, Suganami T, Ogawa Y: Highly purified eicosapentaenoic acid reduces cardio-ankle vascular index in association with decreased serum amyloid A-LDL in metabolic syndrome. Hypertens Res, 2009; 32: 1004-1008

30) Griendling KK, FitzGerald GA: Oxidative stress and cardiovascular injury: Part I: Basic mechanisms and in vivo monitoring of ros. Circulation, 2003; 108: 1912-1916

31) Griendling KK, FitzGerald GA: Oxidative stress and cardiovascular injury: Part II: Animal and human studies. Circulation, 2003; 108: 2034-2040

32) Hiromoto M, Toma $Y$, Tomochika $Y$, Umemoto S, Matsuzaki M: Echographical assessment of the early stage of experimental atherosclerosis of the descending aorta in rabbits. Jpn Circ J, 1996; 60: 691-698

33) Hironaka K, Yano M, Kohno M, Tanigawa T, Obayashi M, Konishi M, Umemoto S, Matsuzaki M: In vivo aortic wall characteristics at the early stage of atherosclerosis in rabbits. Am J Physiol, 1997; 273: H1142-1147

34) Morrow JD, Roberts LJ: The isoprostanes: Unique bioactive products of lipid peroxidation. Prog Lipid Res, 1997; 36: $1-21$

35) Nakamura N, Hamazaki T, Ohta M, Okuda K, Urakaze M, Sawazaki S, Yamazaki K, Satoh A, Temaru R, Ishikura Y, Takata M, Kishida M, Kobayashi M: Joint effects of HMG-CoA reductase inhibitors and eicosapentaenoic acids on serum lipid profile and plasma fatty acid concentrations in patients with hyperlipidemia. Int J Clin Lab Res, 1999; 29: 22-25

36) Burns T, Maciejewski SR, Hamilton WR, Zheng M, Mooss AN, Hilleman DE: Effect of omega-3 fatty acid supplementation on the arachidonic acid: Eicosapentaenoic acid ratio. Pharmacotherapy, 2007; 27: 633-638

37) Miyashita Y, Endo K, Saiki A, Ban N, Yamaguchi T, Kawana H, Nagayama D, Ohira M, Oyama T, Shirai K: Effects of pitavastatin, a 3-hydroxy-3-methylglutaryl coenzyme a reductase inhibitor, on cardio-ankle vascular index in type 2 diabetic patients. J Atheroscler Thromb, 2009; 16: 539-545

38) Vlachopoulos C, Aznaouridis K, O'Rourke MF, Safar ME, Baou K, Stefanadis C: Prediction of cardiovascular events and all-cause mortality with central haemodynamics: A systematic review and meta-analysis. Eur Heart J, 2010; 31: 1865-1871 